\title{
TOO MANY DULL WORDS EXCEED THE LIMITS OF VISUAL PERCEPTION: THE EFFECTS OF CLUTTER AND COLOUR ON LEARNING
}

\author{
Olivia Foulds \\ The University of Strathclyde, United Kingdom
}

\begin{abstract}
When too much visual stimuli is present, the phenomenon of clutter is known to degrade an individual's perception across a variety of domains, ranging from completing search tasks incorrectly, to decreasing reading speed when letters are too close together. However, research is lacking as to whether the negative effects of clutter impact learning when too many words are visible at any one given time. Furthermore, colour has been implicated in affecting clutter. Thus, the present study created a recognition experiment whereby 42 participants had to learn target words that were presented in black or red font and positioned amongst no clutter, clutter words (distractor words surrounded the target), and clutter non-words (sequence of random letters surrounded the target). Results found that words learned in isolation were identified faster and significantly more accurately than words learned in both forms of clutter. Although red target words did not eliminate the negative effects of clutter, red words did show a trend towards higher accuracy of recognition compared to black words. These results would appear to be explained by existing clutter theories that state the limits of attentional resources and short-term memory cannot process excess visual stimuli. These findings have real-world implications for establishing optimal reading formats to improve learning.
\end{abstract}

\section{KEYWORDS}

Clutter, Crowding, Colour, Memory, Reading

\section{INTRODUCTION}

During human development, vision is fundamental to facilitate learning, knowledge, and cognition (Chokron \& Dutton, 2016). However when too much visual stimuli occurs, human cognitive capacity is overloaded. Specifically, the detrimental effect of excess visual stimuli has been defined by the concept of clutter -otherwise known as crowding- which refers to the negative impact of nearby contours that interfere with and reduce visual discrimination when trying to focus on a target (Rosenholtz et al, 2007). Clutter then overloads cognitive resources such as attention and short-term memory, which leads to a bottleneck that impairs object perception (Levi, 2008). Clutter therefore occurs in a multitude of areas, from everyday errands such as finding something in the refrigerator, to essential visual search tasks like $\mathrm{X}$-ray baggage scanning for dangerous items (Adamo et al., 2015). Consequently, it is vital to ensure that when people learn, the amount of visible clutter is minimal.

\subsection{Visual Crowding}

Defining clutter simply as the number of items around a target, many experiments have discovered a similar theme- increasing clutter impairs efficiency of individuals performing search tasks by increasing response times and the number of errors made (Moacdieh and Sarter, 2015, Yeh et al., 2012, Adamo et al., 2015). A recent review on the types of stimuli that have been found to exhibit clutter involve studies that have investigated letters, shapes, objects, digits, abstract symbols, and natural scenes (Patro \& Huckauf, 2019). As clutter has been implicated in affecting letter identification, some studies have tested whether reading words results in more clutter when letters are closer together. Theoretically, by increasing the interletter spacing, each individual letter in a word should appear less crowded and thus enable a more accurate perception of the letter 
resulting in faster reading times (Perea et al., 2011). In a lexical decision task, Perea et al. (2011) confirmed that participants did have faster response times to identifying words or nonsense-words when spacing between letters was increased (Compare casino vs. c a s i n o). However in another lexical decision task, Montani et al. (2015) reported different results: increased letter spacing did not result in statistically significant faster response times, but response times were significantly slower when default letter spacing was reduced. These results would appear to suggest that either readers have adapted to the default presentation of words, or that due to increased spacing, fewer letters can physically be viewed in the visual span thus resulting in longer reading times when letters are further spaced apart (Chung, 2002). Although findings on interletter spacing using lexical decision tasks appear inconclusive, studies using different methodologies have replicated the finding that reduced interletter spacing results in poorer reading fluency. For example, reading times were faster when increased interletter spacing was used in a standardised word reading fluency test (Van-Den-Boer and Hakvoort, 2014). With differing research designs finding complementary results, the validity that clutter of individual letters does impair reading of single words is increased.

However, despite many different types of research design exploring the effects of clutter on interletter spacing, to the best of my knowledge, no studies have yet investigated whether the effects of clutter extend onto more everyday reading scenarios, such as reading words that are surrounded by other words. Yet in the current era of technology, apps have been designed to provide alternative ways to read, such as VelocityReading (2018) and Spritz (2016). These apps display text in a rapid-serial-visual-presentation (RSVP): words do not appear in a block of sentences or paragraphs as they would in a normal book, but instead are quickly, sequentially presented one-by-one. It is believed that using RSVP methods results in faster reading times because an individual's eyes do not have to move (Spritz, 2016). Although one study would appear to confirm that reading using RSVP does result in faster reading speeds than for text that is static- 1171 words were read in a minute of RSVP reading, compared to 303 words for normal text (Rubin and Turano, 1992) - little empirical research evidence explains the mechanisms behind this finding. Additionally, it is unknown as to how well the words that are read at such faster speeds are retained. If the phenomenon of clutter increases errors made in search tasks and increases reading time when letters are too crowded together, perhaps the results of reading through RSVP are also indicative of the phenomenon of clutter: words in a paragraph may be muddling the identification of the specific word that the reader wishes to focus on.

\subsection{Colour}

Generally, the negative perceptual effects of clutter have been shown to increase depending on the similarity of colour, contrast, and visual complexity between the target and clutter that is present (Cheung \& Cheung, 2017). Therefore, when participants were asked to identify a target letter surrounded by other irrelevant letters, the effects of clutter were found to reduce when the target and clutter differed in colour (Põder, 2007). Specifically, Põder's (2007) three experiments demonstrated that when distractor letters were black and the target letter was either red, or highlighted in red or yellow, identification times of the target were significantly faster for all 7 adults that participated. Põder (2007) suggested that these results further accentuated clutter theories which state that excess visual stimuli exceeds the limits of attentional resources- by highlighting a target in a particular colour amongst cluttered distractors of a different colour, exogenously controlled attention was automatically attracted to the salient colour, thus reducing the cluttered illusion from the distractors. Although these experiments have not been replicated and the sample was small, the initial findings from Põder (2007) would appear to suggest that colour does have the ability to affect reading of letters. This is in line with other research irrespective of clutter, that has found faster reading speeds when every alternate word in a sentence was displayed in a different colour (Zhou et al., 2020), or when coloured overlays have been used (Wilkins et al., 1996). Similarly, coloured overlays have been used as a clinical intervention for individuals with dyslexia and autism to improve their attention, which demonstrates the power that colour can have on reading (Wilkins, 2003, cited in Dzulkifli and Mustafar, 2013; Ludlow and Wilkins, 2009).

Related to the theory that clutter doesn't just exceed attentional resources but is also implicated in memory (Levi, 2008), colour has also been identified as impacting upon generalised memory recall. For example, patients with Alzheimer's Disease were presented with a picture such as a purple cow, and later asked to identify what they had seen from a choice of answers such as a pink horse or yellow elephant (Cernin et al., 2003). Results found that participants were significantly better at remembering what they had seen when stimuli were presented in colour compared to black and white. A more recent review on the effect of colour on memory 
reaffirmed these results and concluded that colour can increase memory on certain tasks depending on the choice of colours used (Dzulkifli and Mustafar, 2013). For instance, when recalling letters in an experiment, slides with a white background elicited higher immediate retention rates than slides against a blue and green background (McConnohie, 1999, cited in Dzulkifli and Mustafar, 2013). Furthermore, Greene et al. (1983, cited in Dzulkifli and Mustafar, 2013) explained that colours such as red and orange elicited greater attentional capture compared to colours like brown and grey, and that this was contributing to enhanced memory performance. Despite converging evidence indicating that colour impacts upon memory, little is known as to whether the colour of words affects memory during reading and learning.

\subsection{Summary and Hypotheses}

To summarise, clutter increases response times and the number of errors made in search tasks (Moacdieh and Sarter, 2015), and clutter of individual letters impairs reading speed (Yeh et al. 2012). However, little is known for whether the detrimental effects of clutter extend onto an individual's perception when reading and learning words that are surrounded by other words. In accordance with the measure of clutter proposed by Yu et al. (2014), the present study quantified clutter by the amount of similar image features that merge into a perceptual scene. Thus, when reading words, 'clutter' was defined by multiple irrelevant words and non-words visible on the same screen. It was firstly hypothesised that words learnt amongst either form of clutter would be associated with worse and slower accuracy in a recognition task than words learnt in isolation. Furthermore, as research has identified the colour red as eliciting greater attention, increased object recognition, and faster reading times (Põder, 2007; Dzulkifli and Mustafar, 2013), words that are learnt in red font should hypothetically elicit greater and faster accuracy than words learnt in black font. Finally, since Põder (2007) established that colour interacts with clutter, such that red letters among black distractors reduced the negative perceptual effects of clutter, it was hypothesised that target words learnt in red font would result in similar accuracy and response times to words that were learned in isolation.

\section{METHODOLOGY}

A 3x2 repeated-measures factorial design experiment was programmed and run using OpenSesame (Mathôt et al., 2012). 42 participants ranging from 19 to 78 years old ( 29 female; 13 male; mean age $=42.6$ years) were recruited through a university experimental participation website and completed the study. After a practice task to familiarize users with the system, each participant completed 6 blocks in a randomized order to reduce any order effects from occurring. For each block, 10 target words were sequentially positioned in the centre of the screen for $6000 \mathrm{~ms}$ each, and participants were informed they must try to remember these. Participants were also informed that the word they were to remember would sometimes be presented in isolation whereas sometimes other words would be visible on the same screen, and that they were only to attend to the central word, where a fixation point would direct them to in-between screens. After all 10 target words had appeared, participants underwent a recognition task, where the same target words were randomly interspersed with an additional 20 words, and participants had to individually select whether they had previously seen the word or not. Average reaction times and correct responses for the recognition task in each block were measured as the dependent variables. The system generated answer logs for each participant, which were then transferred into SPSS software for statistical analysis.

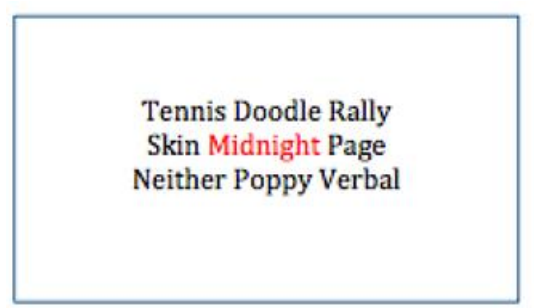

Figure 1. Block 5, where clutter-words are present in the red condition 
The 6 blocks represented the different conditions for the study. Firstly, clutter was defined by what surrounded the target word that participants were instructed to remember: 1) isolation- no other words were around the target; 2) clutter-words- 8 irrelevant words symmetrically surrounded each target (See Figure 1 for an example); and 3) clutter non-words- 8 irrelevant words that did not make sense symmetrically surrounded each target, Eg. 'vinsoi'. Non-words were used to reduce the potential confound that any results found were in fact due to clutter, and not just related to the linguistic structure that distractor words would automatically be read by the reader. Secondly, colour was operationalized so that target words were either presented in 1) black or 2) red font. Between each block, participants were informed that they no longer needed to remember the previous words, and that they could initiate the next block when they were ready.

All stimuli were presented in 32-pt font with default interletter spacing against a white background. Words were selected through generating a list of items on the English Lexicon Project database and chosen to ensure each condition had an equivalent word for: Word length (4-8 letters); number of syllables (1-2); beginning letter; Freq_HAL > 1000; and a I_Mean_RT range of 500-900 (Balota et al., 2007). Plural and distressing words were excluded. The additional words for the recognition task, always presented centrally in black font, were as alike to the target words as possible by sharing the same number of letters, syllables, beginning letter, and frequency range. The non-words were created by rearranging letters from each clutter word, ensuring that each 'non-word' had an equivalent normal word with regards to: beginning letter, word length, syllabic structure, and looked linguistically pronounceable. For example, 'tennis' was changed to 'tesnin'. Both clutter words and non-words always appeared in black font.

Finally, all participants: gave online informed consent; were able to read English; had normal or corrected-to-normal vision by self-report; were naïve to the purpose of the experiment; and received no payment for participation. Overall, the experiment lasted about 20 minutes. Afterwards, a virtual debriefing statement was displayed that thanked individuals for participating, explained the purpose of the experiment, and provided the researcher's contact details for any future concerns they may have had.

\section{RESULTS}

Multiple repeated-measures ANOVAs were conducted to compare the accuracy and response times to identifying target words depending on whether they had been learnt in isolation, amongst clutter-words, amongst clutter-nonwords, or in black or red font.

\subsection{Accuracy}

The results of a $3 \times 2$ repeated measures ANOVA with a Greenhouse-Geisser correction revealed that there was a significant main effect of clutter on participant's accuracy at identifying target words $(\mathrm{F}(1.67,68.28)=9.10$, $\mathrm{p}=.001$, $\mathrm{np} 2=.182$ ). Bonferroni Post Hoc tests confirmed that mean accuracy scores were significantly higher when identifying target words that had been presented in no clutter (mean $=8.92$ ) compared to target words that had been presented among clutter words (mean=7.93) and clutter non-words (mean=8.13). The difference between accuracy on clutter words and clutter non-words did not reach significance.

While descriptive statistics revealed that participant's mean accuracy scores were slightly higher when verifying red target words (mean=8.49) compared to black target words (mean=8.16), the ANOVA with sphericity assumed revealed that this difference was not significant $(\mathrm{F}(1,41)=3.93, \mathrm{p}=.054, \mathrm{np} 2=.087)$.

Furthermore, there was no significant interaction between clutter and colour $(\mathrm{F}(2,82)=1.07, \mathrm{p}=.346$, $\mathrm{np} 2=.026$ ). However, for each clutter condition, participants did have slightly higher accuracy when the target word had been initially presented in red as opposed to black font (See Table 1 and Figure 2a). 
Table 1. Descriptive statistics (Mean \pm Standard Deviation) for: Accuracy as the number of correctly identified target words per condition; and Reaction times to identifying target words

\begin{tabular}{|l|l|l|l|l|}
\hline & \multicolumn{2}{|c|}{ Accuracy } & \multicolumn{2}{c|}{ Reaction time (ms) } \\
\hline & \multicolumn{2}{|c|}{ Target Word Colour } & \multicolumn{2}{c|}{ Target Word Colour } \\
\hline Clutter Type & Black & Red & Black & Red \\
\hline Isolation & $8.76 \pm 1.66$ & $9.07 \pm 1.13$ & $902.96 \pm 517.82$ & $871.54 \pm 418.92$ \\
\hline Clutter words & $7.62 \pm 2.00$ & $8.24 \pm 2.26$ & $947.54 \pm 624.94$ & $973.26 \pm 745.75$ \\
\hline Clutter non-words & $8.10 \pm 1.82$ & $8.17 \pm 1.85$ & $942.98 \pm 577.62$ & $938.67 \pm 627.65$ \\
\hline
\end{tabular}

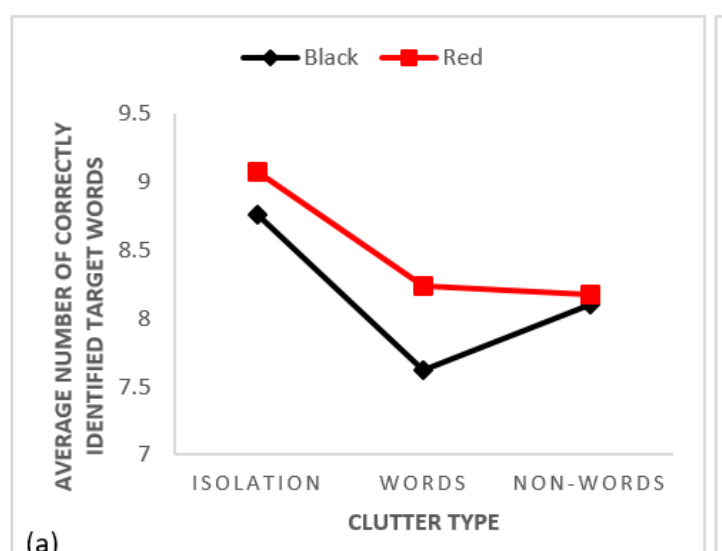

(a)

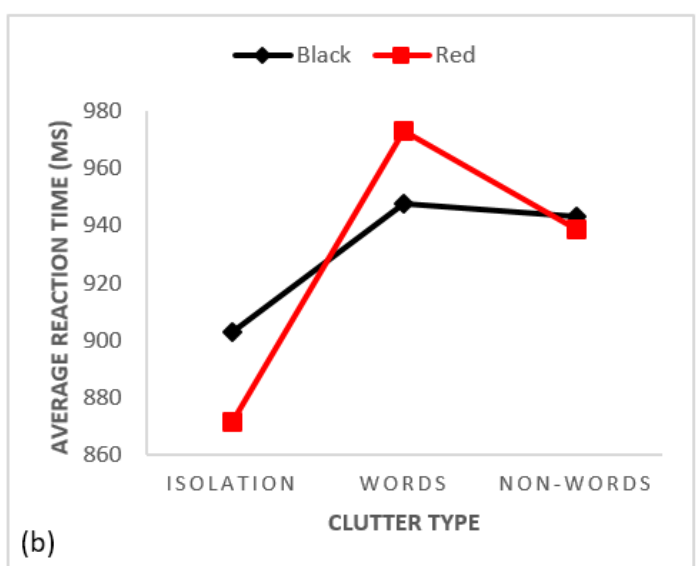

(b)

Figure 2. Mean results for each condition, where black and red refer to the target word colour: (a) Accuracy for the number of correctly identified target words; (b) Reaction times for target word responses

\subsection{Average Reaction Time}

A repeated measures ANOVA with Greenhouse-Geisser correction identified a significant main effect of clutter on reaction time $(\mathrm{F}(1.94,2384.28)=11.35, \mathrm{p}=.000, \mathrm{np} 2=.009)$. Bonferroni Post Hoc tests revealed that reaction times to target words presented in no clutter (mean $=887.25 \mathrm{~ms}$ ) were significantly faster than both clutter words (mean $=960.60 \mathrm{~ms}$ ) and clutter non-words (mean $=940.82 \mathrm{~ms}$ ).

Although reaction times for the red words were slightly faster $(927.82 \mathrm{~ms})$ than the black words $(931.29 \mathrm{~ms})$ this result did not reach significance $(\mathrm{F}(1,1229)=.07, \mathrm{p}=.789, \mathrm{np} 2=.000)$.

Furthermore, a visible interaction occurred between colour and clutter (See Table 1 and Figure 2b): When there was no clutter or clutter non-words, participants responded slightly faster to red words than black words. Yet when clutter words were present, participants responded slightly faster to black words than red words. However, this interaction did not reach significance $(\mathrm{F}(1.99,2440.43)=1.82, \mathrm{p}=.162, \mathrm{np} 2=.001)$.

\section{DISCUSSION}

As hypothesised, words that were learned in clutter elicited significantly impaired accuracy and longer reaction times when recalling them in a recognition task compared to words that were learnt in isolation. Additionally, despite the hypothesis that red target words would result in greater accuracy and faster reaction times compared to black target words, no significant differences were found. However, a trend did appear that red target words elicited higher recognition accuracy and were recalled slightly faster than black words. Furthermore, whilst it was predicted that red target words presented amongst black clutter might reduce the negative effects of clutter and lead to similar accuracy and reaction times to words that had been learned in isolation, there was no significant interaction found between clutter and colour. 
The finding that clutter around a target word, either in the form of irrelevant words or non-words, increased the number of errors made and reaction times in response to a recognition task, would appear consistent with a multitude of previous research that identified clutter as degrading accuracy and response times in search tasks (Moacdieh and Sarter, 2015; Yeh et al., 2012; Adamo et al., 2015). Additionally, the present findings assimilate to faster reading times when the clutter surrounding an individual letter in a word was reduced (Perea et al., 2011; Van-den-Boer \& Hakvoort, 2014). As the present study found a significant impairment of target word recognition when the word had been learned in clutter as opposed to isolation, the most reasonable conclusion for these results would appear to relate to other study's explanations of clutter- negative perceptual effects of clutter do extend onto more everyday reading scenarios, such as words surrounding other words: clutter automatically attracts vision away from the target and this excess of visual stimuli exceeds the limits of attentional resources and short-term memory (Levi, 2008). For example, when participants are attempting to memorise a target word, if other words or non-words are surrounding the target, these may be mistakenly processed as the target word and cause a degradation of memory because only a certain number of items can be processed at one time (Chastain, 1991). Furthermore, an interesting finding with the present study is that the participant's attention was not automatically directed towards the excess clutter because they were not expecting it, as participants were explicitly pre-warned that they did not need to look at the words beyond the center of the screen. Therefore, it is unknown whether the effects of clutter are so strong that they overrule overt attention and distract a user's vision regardless of whether they want to process it or not, or whether the effects may be a result of more subconscious and covert behavior that participants are unaware of, perhaps through their peripheral vision subtly processing the excess stimuli. Consequently more research is needed, perhaps using eye-tracking, to explain how exactly visual attention responds to clutter during reading.

In keeping with theories related to attention, colour has previously been thought to capture attention and thus enhance memory performance (Dzulkifli and Mustafar, 2013). Although the present study did not find a significant effect of using the colour red, compared to black, on accuracy of identifying target words in a recognition task, this finding only marginally missed out on reaching significance $(p=.054)$. Therefore, it could be tentatively assumed that a larger sample size may have resulted in a significant result that words learned in red font do in fact elicit better recognition than black. This explanation would reiterate the findings by Greene et al. (1983, cited in Dzulkifli and Mustafar, 2013), who stated that red elicited greater attentional capture compared to colours like brown and grey, and that this impacted upon memory performance. However, other research has claimed that long wavelength colours like red illicit worse performance in certain tasks (Shieh and Lin, 2000; Hall and Hanna, 2004). Furthermore, unlike research that identified colour as increasing reading speeds (Wilkins et al., 1996) the present study found minimal differences in response times for either red or black target words. Thus, further research is needed to clarify the contrasting results of whether the colour red enhances or hinders individual's performance in tasks.

It is unsurprising that as the present study found no significant benefit of presenting the target word in red, compared to black, red target words also failed to reduce the negative effects of surrounding clutter. These results are in contrast to Põder's (2007) experiments that found when target and surrounding clutter differed in colour, the process of mentally eliminating distractors was easier (Levi, 2008). However, these findings should not automatically reject the theory that colour can reduce negative effects of clutter because: only two colours were used-red and black; participants were not screened for colour-blindness; and due to variations in computer browser that the experiment was viewed on, the brightness could not be controlled for across participants. Additional examination using different methods would be useful to clarify these methodological problems of how the colour red contrasted to the colour black.

The results of the present experiment are not just theoretical, but could also have vital importance in many aspects of everyday life because the finding that clutter does impair individual's performance across a multitude of tasks has been replicated. More specifically, the present study provided the first evidence (to the best of our awareness) that the negative perceptual effects of clutter extend onto reading words surrounded by other words. As this is how many textbooks and websites present information, the present experimental findings could evolve current learning strategies and alter popular opinion on how best to read and retain words. With vision being a huge facilitator of learning (Chokron \& Dutton, 2016), it may be a more positive and beneficial learning experience if clutter is considered for future design of learning materials. Therefore apps that operate rapid-serial-visual-presentation, or handheld devices like typoscopes which ensure only a few words are visible at any one time, could potentially offer a more optimal reading environment in comparison to a standard book. 
This alternative way to read should then hypothetically promote greater memory of what has been read, and in a faster time-period. Presenting words in a minimalistic structure may also enable more inclusive education to occur, as other research has identified that children with cerebral visual impairment or dyslexia particularly struggle with clutter (Chokron \& Dutton, 2016; Morris et al., 2018).

However, it is worth noting some limitations of the present study. Firstly, in contrast to clutter studies conducted in a controlled laboratory, anyone with OpenSesame could participate in the experiment. Therefore, although all words were presented in a consistent size, due to variation among computers and distance from the screen, some participants would have seen the words in different sizes, resolutions, and colour contrasts. Other studies on clutter have removed this confound and ensured a fixed viewing distance by using a chin rest (Yeh et al., 2012; Adamo et al., 2015). However, this may not be necessary when other research has concluded that clutter perception is invariant to image size, but dependent on the content (Zelinsky and Yu, 2015). Furthermore, as the experiment was conducted virtually, it was unknown whether participants varied in terms of factors that may affect their memory such as: their mood; the time of day the experiment was completed; distractions that were present; and alcohol or drug consumption. Nonetheless, these limitations could in fact be seen as a positive- no data was excluded from analyses, yet results were still significant. Therefore, the overall finding that clutter does significantly impair word recognition may extend onto a population irrelevant of individual situation or distractions present.

In order to fully establish whether the effects of clutter similarly extend onto other populations, further research would firstly be needed. In particular, it would appear worthwhile to examine whether the degradation of word recognition among other distractor words exists among children- after all, it is children who are expected to memorise what they learn through textbooks and websites that contain a plethora of clutter. It may be possible that children are unaffected by clutter, and that it is a concept that develops and worsens with age. Alternatively, it is unknown whether the negative effects of clutter can be alleviated through practice, and therefore reading words surrounded by other words in a normal paragraph do not result in clutter effects if readers are familiar with reading in that way. Correspondingly, as the present experiment only tested recognition of individual words, it would be interesting to expand the ecological validity and see whether clutter affects word recognition at a sentence level. Furthermore, future research should develop the design of the current experiment to explore whether learning words amongst clutter impairs memory recognition beyond the immediate short-term memory, and not just instantly after words have been presented. Consequently, the present research is only the beginning of a topic that could potentially improve learning in many domains. All of these unanswered questions therefore raise many interesting avenues for future research that could have hugely important real-life consequences for how best to learn when clutter is present. If more understanding of clutter is had, then hopefully strategies can be implemented to reduce the negative effects it causes.

\section{CONCLUSION}

To summarise, the current experiment identified that clutter, either in the form of distractor words or non-words, significantly impaired accuracy and increased response times for recognising target words. This finding would appear to extend other theories that when excess visual stimuli are present, the limits of short-term memory and attention are exceeded. Although no significant findings were found in relation to the colour of a target word or the interaction between clutter and colour, some interesting patterns and trends emerged that require more investigation. Finally, as this experiment provides the first evidence that clutter effects do extend onto words surrounding other words, these findings motivate further research for creating an optimal reading format for learning. For example, if clutter can be reduced during reading, through ways such as apps that operate rapid-serial-visual-presentation, then reading speed should be faster while improving memory for what has been seen.

\section{ACKNOWLEDGEMENT}

This paper was revised from work undertaken as part of the author's undergraduate degree with The Open University. The author would like to thank their tutor at the time, M Louise Kelly, for their original feedback. Additionally, appreciation goes to the anonymous reviewers for providing helpful suggestions. 


\section{REFERENCES}

Adamo, S., Cain, M., \& Mitroff, S. (2015) 'Targets Need Their Own Personal Space: Effects of Clutter on Multiple-Target Search Accuracy', Perception, vol. 44, no. 10, pp. 1203-1214.

Balota, D.A., Yap, M.J., Cortese, M.J., Hutchison, K.A., Kessler, B., \& Loftis, B. (2007) 'The English Lexicon Project', Behavior Research Methods, vol. 39, no. 3, pp. 445-59.

Cernin, P., Keller, B., \& Stoner, J. (2003) 'Color Vision in Alzheimer's Patients: Can We Improve Object Recognition With Color Cues?', Aging, Neuropsychology, and Cognition, vol. 10, no. 4, pp. 255-267.

Chastain, G. (1991) 'Effects of abruptly appearing clutter on a peripherally precede covert attention shift', The Journal of General Psychology, vol. 118, no. 1, pp. 31-44.

Cheung, L. \& Cheung, S. (2017) 'Chinese-character crowding-Effects of structural similarity', Journal of Vision, vol. 17, no. 11 , pp. 1-13.

Chokron, S., \& Dutton, G. (2016) 'Impact of Cerebral Visual Impairments on Motor Skills: Implications for Developmental Coordination Disorders', Frontiers In Psychology, vol. 7, no. 1.

Chung, S. (2002) 'The effect of letter spacing on reading speed in central and peripheral vision', Investigative Ophthalmology \& Visual Science, vol. 43, no. 4, pp. 1270-1276.

Dzulkifli, M., \& Mustafar, M. (2013) 'The Influence of Colour on Memory Performance: A Review', Malaysian Journal of Medical Sciences, vol. 20, no. 2, pp. 3-9.

Hall, R., \& Hanna, P. (2004) 'The impact of web page text-background colour combinations on readability, retention, aesthetics and behavioural intention', Behaviour and information technology, vol. 23, no. 3, pp. 183-195.

Levi, D. (2008) 'Crowding_An essential bottleneck for object recognition: A mini-review', Vision Research, vol.48, no. 5, pp. 635-654.

Ludlow, A. \& Wilkins, A. (2009) 'Case report: color as a therapeutic intervention', Journal of Autism and Developmental Disorders, vol. 39, no. 5, pp. 815-818.

Mathôt, S., Schreij, D., \& Theeuwes, J. (2012) 'OpenSesame: An open-source, graphical experiment builder for the social sciences', Behavior Research Methods, vol.44, no.2, pp.314-324 [Computer Program]. Available at: https://www.ncbi.nlm.nih.gov/pubmed/22083660

Moacdieh, N., \& Sarter, N. (2015) 'Clutter in Electronic Medical Records, Examining its performance and attentional costs using eye tracking', Human Factors: The Journal of Human Factors and Ergonomics Society, vol. 57, no. 4, pp. 591-606.

Montani, V., Facoetti, A., \& Zorzi, M. (2015) 'The effect of decreased interletter spacing on orthographic processing', Psychonomic Bulletin \& Review, vol. 22, no. 3, pp. 824-832.

Morris, M.R., Fourney, A., Ali, A. and Vonessen, L. (2018) 'Understanding the needs of searchers with dyslexia', In Proceedings of the 2018 CHI Conference on Human Factors in Computing Systems (pp. 1-12).

Patro, K., \& Huckauf, A. (2019) 'Asymmetries in flanker-target interference at different levels of number processing', Acta psychologica, vol. 201.

Perea, M., Moret-Tatay, C., \& Gómez, P. (2011) 'The effects of interletter spacing in visual-word recognition', Acta Psychologica, vol. 137, no. 3, pp. 345-351.

Põder, E. (2007) 'Effect of colour pop-out on the recognition of letters in crowding conditions', Psychological Research, vol.71, no.6, pp.641-645.

Rosenholtz, R., Li, Y., \& Nakano, L. (2007) 'Measuring visual clutter', Journal of Vision, vol.7, no.2.

Rubin, G. \& Turano, K. (1992) 'Reading without saccadic eye movements', Vision Research, vol. 32, no. 5, pp. 895-902.

Shieh, K. \& Lin, C. (2000) 'Effects of screen type, ambient illumination, and color combination on VDT visual performance and subjective preference', International Journal of Industrial Ergonomics, vol. 26, no. 5, pp. 527-536.

Spritz (2016) 'Spritz' [Computer application]. Available at: http://spritzinc.com

Van-Den-Boer, M., \& Hakvoort, B. (2014) 'Default spacing is the optimal spacing for word reading', The Quarterly Journal of Experimental Psychology, vol. 68, no. 4, pp. 697-709.

VelocityReading (2018) 'Velocity Reading' [Computer application]. Available at: https://velocityreading.com/learn

Wilkins, A., Jeanes, R., Pumfrey, P., \& Laskier, M. (1996) 'Rate of Reading Test $\AA$ : Its reliability, and its validity in the assessment of the effects of coloured overlays', Ophthalmic and Physiological Optics, vol. 16, no. 6, pp. 491-497.

Yeh, S., He, S., \& Cavanagh, P. (2012) 'Semantic Priming From Crowded Words', Psychological Science, vol. 23, no. 6, pp. 608-616.

Yu, C., Samaras, D., \& Zelinsky, G. (2014) 'Modeling visual clutter perception using proto-object segmentation', Journal of Vision, vol. 14, no. 7.

Zelinsky, G., \& Yu, C. (2015) 'Clutter perception is invariant to image size', Vision Research, vol. 116, pp. 142-151.

Zhou, W., Ye, W., \& Yan, M. (2020) 'Alternating-color words facilitate reading and eye movements among second-language learners of Chinese', Applied Psycholinguistics, vol. 41, no. 3, pp. 685-699. 